\title{
TERAPIA DE CONSCIÊNCIA CORPORAL EM MULHERES PÓS-CIRURGIA DE CÂNCER DE MAMA
}

\author{
Lara Nery Peixoto ${ }^{1}$, Fernanda Stellutti Magrini Pachioni ${ }^{2}$, Elisa Bizetti Pelai ${ }^{2}$, Alessandra Madia \\ Mantovani ${ }^{4}$, Mariana de Carvalho Pinto ${ }^{2}$, Mariana Romanholi Palma ${ }^{2}$, Edna Maria do Carmo ${ }^{3}$, \\ Cristina Elena Prado Teles Fregonesi ${ }^{2}$
}

\footnotetext{
${ }^{1}$ Universidade Estadual Paulista - UNESP, Faculdade de Ciências e Tecnologia, Pós-Graduação Lato Sensu em Fisioterapia, ${ }^{2}$ Curso de Mestrado em Fisioterapia, ${ }^{3}$ Departamento de Fisioterapia, Presidente Prudente, SP; ${ }^{4}$ UNESP, Instituto de Biociências, Programa de Pós-Graduação Strictu Sensu, Rio Claro, SP. e-mail: larinhapeixoto@hotmail.com
}

\section{RESUMO}

O estudo objetivou analisar o efeito da terapia de consciência corporal (TCC) na qualidade de vida (QV), na ansiedade e na depressão de mulheres após a cirurgia de retirada do câncer de mama. Participaram mulheres submetidas a tratamento cirúrgico de câncer de mama. Todas as participantes foram submetidas a uma avaliação da qualidade de vida (Study's Short Form-36 - SF36), da ansiedade e depressão (escala Hospital Anxiety and Depression Scale - HAD) antes e após realizarem 16 sessões de terapia de consciência corporal, em grupo, com duração de 60 minutos, com a frequência de duas vezes por semana. As sessões eram divididas em inicio da consciência corporal, movimentos específicos associados à respiração, exercícios e relaxamento consciente. Houve melhora na ansiedade, depressão e QV, sendo significativa nos domínios capacidade funcional, aspectos físicos, dor, estado geral de saúde, aspectos sociais e aspectos emocionais. Concluiu-se que a TCC é efetiva na melhora da QV, na ansiedade e na depressão dessa população, mas faz-se necessário um estudo com um número maior de indivíduos para verificar a reprodutibilidade dos referidos achados.

Palavras-chave: reabilitação, qualidade de vida, ansiedade, depressão.

\section{BODY AWARENESS THERAPY IN WOMEN AFTER SURGERY FOR BREAST CANCER}

\begin{abstract}
The objective of this study was to analyze the effect of body awareness therapy (BAT), on quality of life (QOL), anxiety and depression in women after surgical removal of breast cancer. Participated women in undergoing surgical treatment of breast cancer. All participants were subjected to evaluation of quality of life (Study's Short Form-36 - SF-36), anxiety and depression (Hospital Anxiety and Depression Scale - HAD) before and after undergoing 16 sessions of therapy body awareness, group, lasting 60 minutes, with the frequency twice a week. The sessions were divided into early body awareness, specific movements associated with breathing exercises and conscious relaxation. It was observed a significant improvement in anxiety, depression and QOL, mainly on fields' physical functioning, role physical, pain, vitality, social and emotional aspects. We conclude that BAT is effective in improving QOL, anxiety and depression in this population, but it is necessary a study with a larger number of individuals to check the reproducibility of these findings.
\end{abstract}

Keywords: rehabilitation, quality of life, anxiety, depression. 


\section{INTRODUÇÃO}

Atualmente, o câncer de mama tornou-se uma das grandes preocupações dos profissionais da área da saúde da mulher, em razão da elevada incidência que apresentou na última década, principalmente pelos impactos físicos, psicológicos e sociais que acarreta ${ }^{1}$. Viver com uma doença que impõe características de permanência prolongada, conviver com a possibilidade de recidiva do câncer ${ }^{1}$, bem como com intervenções traumáticas que acarretam em complicações pós operatórias ${ }^{2,3}$, constituemse em algumas das frequentes dificuldades enfrentadas no cotidiano dessas mulheres, favorecendo a desestruturação do seu aspecto biopsicossocial, podendo afetar sua qualidade de vida (QV), o que se torna uma importante questão de saúde pessoal e pública $^{3-5}$.

Nesse contexto, a reabilitação após o procedimento cirúrgico de câncer de mama deve ultrapassar as barreiras das limitações físicas, visto que o aspecto emocional influencia diretamente na progressão do quadro clínico ${ }^{6}$, expondo os envolvidos a uma constante situação de estresse, associada à baixa autoestima, depressão e ansiedade ${ }^{7,8}$.

Visando maior globalidade, a melhora da consciência corporal tem sido descrita como um elemento chave para abordagens terapêuticas, frequentemente classificadas como terapias corpo-mente, com benefícios relatados em uma variedade de enfermidades ${ }^{9}$. Assim, alguns autores têm optado por intervenções focadas na consciência corporal, para melhorar sequelas físicas e psicológicas decorrentes dos tratamentos do câncer de mama, tendo obtido resultados satisfatórios para essa população ${ }^{10}$.

Dentre as intervenções corpo-mente está a Terapia de Consciência Corporal (TCC), desenvolvida pelo psicanalista francês Dropsy $^{11}$ e previamente descrita na fisioterapia psiquiátrica pelo fisioterapeuta Roxendal, na década de 1980. A TCC utiliza de movimentos, respiração e consciência para tentar restaurar o equilíbrio entre o corpo e a mente ${ }^{12}$. Apesar de estudos demonstrarem os benefícios dessa terapia em diversas enfermidades ${ }^{13-15}$, não há evidências científicas que demonstrem a aplicação da TCC em mulheres após a incidência de câncer de mama.

Sendo assim, a comprovação científica da TCC ainda é fundamental para sua utilização como alternativa de tratamento, uma vez que as evidências do seu efeito são escassas. Portanto, o presente estudo teve por objetivo verificar a eficácia da TCC na qualidade de vida, na ansiedade e na depressão de mulheres após o procedimento cirúrgico de retirada do câncer de mama. 
MATERIAS E MÉTODOS

Participaram quatro mulheres submetidas a tratamento cirúrgico de câncer de mama, independente do tipo de cirurgia, entre zero a 10 anos de pós-operatório. Foram critérios de inclusão: pacientes com consentimento médico, sem metástase diagnosticada e que não estivessem realizando quimioterapia ou radioterapia. Os procedimentos realizados foram aprovados pelo Comitê de Ética em Pesquisa da Instituição (CAAE: 03195912.7.0000.5402) e todas, após concordarem com os objetivos e procedimentos do estudo, assinaram um Termo de Consentimento Livre e Esclarecido.

Todas as participantes foram submetidas a uma avaliação antes e após realizarem 16 sessões de terapia de consciência corporal, em grupo, com duração de 60 minutos, com a frequência de duas vezes por semana. Foram coletados os dados pessoais e realizadas avaliações da QV e da ansiedade e depressão.

Para avaliação da QV foi utilizado o questionário de qualidade de vida SF-36 Study's Short Form-36 (SF-36), traduzido e validado para a Língua Portuguesa por Ciconelli ${ }^{16}$. É um instrumento composto por 36 itens que avaliam oito domínios: capacidade funcional, aspectos físicos, dor, estado geral de saúde, vitalidade, aspectos sociais, aspectos emocionais e saúde mental.
Cada domínio tem um escore que varia de zero a cem, correspondente ao pior e melhor estado de saúde, respectivamente ${ }^{16}$.

Para avaliar a ansiedade e a depressão foi utilizada a escala Hospital Anxiety and Depression Scale (HAD). Tal escala é composta por 14 itens de múltipla escolha, sendo sete itens referentes à subescala de depressão e sete à de ansiedade. Cada questão pode receber de 0 a 3 pontos, assim a pontuação global de cada subescala pode variar de 0 a 21 . Valor igual ou maior a 9 pontos, em cada subescala, é indicativo de ansiedade e/ou depressão ${ }^{17}$.

As vivências de consciência corporal foram conduzidas por uma fisioterapeuta e levavam a participante a entrar em contato com do seu próprio corpo. A forma pausada de condução das aulas e o silêncio proporcionam ao participante maior contato com o seu próprio corpo percebendo as partes do seu corpo e, com estas se relacionarem. Devido a esse fato, em algumas vivências eram repetidos alguns movimentos várias vezes, de forma lenta, chamando atenção para o movimento executado.

A condução verbal sempre seguiu o sentido do eixo central corporal dos pés para cabeça ou da cabeça para os pés e depois os membros superiores. 
A proposta teve como objetivo proporcionar organização corpórea mais global e equilibrada, sendo então uma organização mais funcional. Foram utilizados recursos terapêuticos auxiliares como colchonetes, bolinhas de tênis, cunha de espuma, espaguete de piscina, bolas de borracha, bolas suíças, disco de propriocepção e equilíbrio, entre outros. Os recursos utilizados tinham o objetivo de chamar a atenção para um local preciso, obtendo-se mais percepção da região solicitada.

As sessões foram iniciadas com um momento para cada participante entrar em contato com seu corpo e iniciar a consciência corporal. No segundo momento foram realizados movimentos voltados a estimular a ativação sensorial com ênfase na postura, marcha, posicionamento do tronco e a manutenção do equilíbrio relacionado com o eixo central do corpo vertical. Ao longo da sessão, a técnica de respiração diafragmática era enfatizada e, todos os movimentos foram ensinados e praticados com padrões respiratórios integrados. A terceira parte das sessões foi dedicada a exercícios destinados a promover a conscientização da tensão e relaxamento muscular, melhorar a coordenação e alternância entre atividade, descanso e relaxamento consciente en para realizar movimentos "eficientes". Por fim, breves momentos de compartilhamento, quando cada participante podia relatar suas sensações corporais e seus sentimentos durante a vivência.

\section{Análise estatística}

A análise do SF-36 foi realizada por meio de análise de contingência pelo teste qui-quadrado, enquanto a caracterização da amostra e a análise da escala HAD foram realizadas descritivamente. Os testes estatísticos foram aplicados por meio do software Graph Pad Prisma 5.0 e padronizou-se o nível de significância de 5\%.

\section{RESULTADOS}

Todas as participantes tinham sido submetidas à cirurgia para remoção do câncer na mama direita, com tempo médio de cirurgia de 57,60 $\pm 36,17$ meses e idade média de $50,75 \pm 3,11$ anos.

O SF-36 evidenciou melhora significativa na maioria dos domínios, com exceção dos domínios vitalidade e saúde mental (Tabela 1). Os resultados da escala HAD demonstraram presença de ansiedade em duas pacientes, sendo que uma delas também apresentava depressão. Após a intervenção, não foi observada ansiedade e depressão nas participantes (Tabela 2). 
Tabela 1. Valores de qualidade de vida obtidos a partir do SF-36. Apresentação da média \pm desvio padrão ( $n=4)$, diferença qui-quadrada (Dif.) e $p$-valor.

\begin{tabular}{lcccc}
\hline Domínios & Antes & Depois & Dif. & p-valor \\
\hline Capacidade funcional & $51,25 \pm 23,6$ & $61,25 \pm 32,1$ & 11,63 & 0,0088 \\
Aspectos físicos & $18,75 \pm 20,7$ & $68,75 \pm 41,0$ & 51,09 & 0,0001 \\
Dor & $44,75 \pm 28,5$ & $55,75 \pm 20,2$ & 44,40 & 0,0001 \\
Estado geral de saúde & $61,50 \pm 23,2$ & $77,75 \pm 15,1$ & 16,72 & 0,00008 \\
Vitalidade & $40,00 \pm 20,3$ & $55,00 \pm 25,2$ & 0,428 & 0,93661 \\
Aspectos sociais & $42,50 \pm 37,7$ & $59,38 \pm 42,7$ & 11,29 & 0,0103 \\
Aspectos emocionais & $41,65 \pm 43,3$ & $66,65 \pm 40,8$ & 50,76 & 0,0001 \\
Saúde mental & $48,00 \pm 24,5$ & $57,00 \pm 27,5$ & 0,394 & 0,9415 \\
\hline
\end{tabular}

Tabela 2. Valores da escala de ansiedade e depressão HAD* antes e após intervenção de cada participante $(n=4)$.

\begin{tabular}{ccccc}
\hline & \multicolumn{2}{c}{ Ansiedade } & \multicolumn{2}{c}{ Depressão } \\
\hline Pacientes & Antes & Depois & Antes & Depois \\
\hline 1 & 6 & 5 & 8 & 7 \\
2 & 1 & 0 & 0 & 0 \\
3 & 16 & 8 & 12 & 8 \\
4 & 10 & 7 & 8 & 5 \\
\hline
\end{tabular}

*Pontuação: subescalas 0-21 com escores igual ou maior a 9 indicam ansiedade e/ou depressão.

\section{DISCUSSÃO}

O presente estudo se propôs a investigar o efeito da intervenção com TCC na qualidade de vida, ansiedade e depressão, em mulheres submetidas à remoção cirúrgica de câncer de mama. Notou-se que tal intervenção foi eficiente, com valores significantes em vários domínios da QV.

Com relação aos resultados do SF-36, observou-se aumento do escore em todos os domínios, sendo este significativo na capacidade funcional, aspectos físicos, dor, estado geral de saúde, aspectos sociais e aspectos emocionais. Uma hipótese para essa melhora significativa tanto nos aspectos físicos como nos psicológicos pode ser, além da eficácia da TCC, os benefícios da interação grupal, principalmente nos aspectos emocionais e sociais, pois proporciona troca de experiências e incentivo da busca pela recuperação.

$\mathrm{Na}$ avaliação inicial, maiores comprometimentos foram observados nos domínios limitação por aspectos físicos, dor e vitalidade, sendo os domínios estado geral de saúde e capacidade funcional menos comprometidos. Outros estudos que avaliaram a qualidade de vida em mulheres com câncer de mama ${ }^{2,3}$, também evidenciaram maior comprometimento nos domínios de limitação por aspectos físicos, dor e vitalidade, porém os autores ainda observaram menor impacto em aspectos emocionais e sociais, o que não foi encontrado nesse estudo já que estes domínios mostraram grande comprometimento. 
A TCC é um tratamento frequente nos países nórdicos, mas poucos estudos avaliando seus efeitos foram publicados. Um estudo comparando a TCC com outras abordagens fisioterapêuticas, por meio do SF-36, avaliou fatores relacionados à saúde em indivíduos com distúrbios osteomusculares não específicos. Neste estudo foi verificada melhora significativa em todos os domínios, sendo mais acentuadas nos domínios de dor, saúde mental, vitalidade e estado geral de saúde e menor significância em aspectos emocionais e sociais $^{13}$, verificando algumas compatibilidades com o presente estudo.

$\mathrm{Na}$ avaliação inicial duas participantes apresentaram ansiedade, sendo que uma também apresentava depressão. Após as 16 sessões de intervenção com TCC, houve melhora nesses aspectos, sendo que nenhuma delas apresentou algum nível de ansiedade ou depressão. Um estudo comparando a TCC com outras terapias para verificar sua eficácia em sintomas psicológicos de indivíduos com distúrbios osteomusculares não específicos mostrou melhora significativa na ansiedade e depressão, tanto na TCC como nas outras terapias avaliadas ${ }^{14}$.

O estudo de Gard $^{16}$ traz um levantamento bibliográfico dos resultados da TCC em várias doenças, mostrando que tratamentos incluindo a TCC melhoraram a QV e a ansiedade em indivíduos com distúrbios osteomusculares prolongados. Mostrou, também, que a TCC apresentou melhores resultados na dor, imagem corporal, QV e sintomas psicológicos, como ansiedade e depressão, quando comparada com outras terapias, em pessoas com distúrbios osteomusculares não específicos ${ }^{15}$. Ademais estudos mostram eficácia da TCC em indivíduos com distúrbios psiquiátricos como distúrbios de personalidade grave, esquizofrenia e depressão, apresentando melhora na QV, na ansiedade e na depressão ${ }^{18-20}$. Portanto, considerando o impacto psíquico advindo do pós-operatório de câncer de mama, é importante investigar a eficácia da intervenção com TCC para essas mulheres, buscando novas técnicas para melhorar a qualidade de vida e sintomas psicológicos nessa população.

Entretanto, devido ao tamanho da amostra no presente estudo, os resultados devem ser interpretados com cautela, pois o número baixo de indivíduos pode ter resultado em subestimação ou superestimação do valor clínico desta terapia. Portanto, faz-se necessário verificar o efeito da TCC em um número maior de indivíduos para obter resultados mais significativos. 


\section{CONCLUSÃO}

Concluiu-se que a participação em um grupo de TCC resultou em melhora da QV e da ansiedade e depressão em mulheres no pós-operatório do câncer de mama, mostrando a importância dessa terapia, visto que atualmente é necessário intervenções mais globalizadas, que possam melhorar ou amenizar as repercussões que o câncer de mama acarreta nessa população.

\section{CONFLITO DE INTERESSES}

Os autores declaram não haver qualquer potencial conflito de interesse que possa interferir na imparcialidade deste trabalho científico.

\section{REFERÊNCIAS}

1. Azevedo RF, Lopes RLM. Concepção de corpo em Merleau-Ponty e mulheres mastectomizadas. Rev Bras Enferm. 2010;63(6):1067-1070. DOI: http://dx.doi.org/10.1590/S0034$\underline{71672010000600031}$

2. Lahoz MA, Nyssen SM, Correia GN, Garcia APU, Driusso P. Capacidade Funcional e Qualidade de Vida em Mulheres PósMastectomizadas. Rev Bras Cancerol. 2010; $4(56): 423-430$.

3. Pelai EB, Figueira JIJ, Mantovani AM, Gomes PRL, Martinelli AR, Fregonesi CEPT et al. Qualidade de vida, depressão e dor em mulheres pós-cirurgia de câncer de mama. Ter Manual. 2012;10(48):12-17.
4. Bertan, FC, Castro, EK. Qualidade de vida e câncer: revisão sistemática de artigos brasileiros. Rev Psicol. 2009;40(3):366-372.

5. Hartl K, Schennach R, Muller M, Engel J, Reinecker $\mathrm{H}$, Sommer $\mathrm{H}$ et al. Quality of life, anxiety, and oncological factors: a follow-up study of breast cancer patients. Psychosomatics. 2010;51(2):112-

123. DOI: http://dx.doi.org/10.1016/S00333182(10)70671-X

6. Alves PC, Silva APS, Santos MCL, Fernandes AFC. Conhecimento e expectativa de mulheres no pré-operatório da mastectomia. Rev Esc Enferm USP. 2010;44(4):989-995. DOI: $\quad$ http://dx.doi.org/10.1590/S0080$\underline{62342010000400019}$

7. Moura FMJSP, Silva MG, Oliveira SC, Moura LJSP. Os sentimentos das mulheres Pós-mactectomizadas. Esc Anna Nery Rev Enferm 2010;14(3):477-484. DOI: http://dx.doi.org/10.1590/S141481452010000300007

8. Arroyo JMG, López MLD. Psychological Problems Derived from Mastectomy: A Qualitative Study. Int J Surg Oncol. 2011;132:461.

9. Mehling WE, Wrubel J, Daubenmier JJ, Price CJ, Kerr CE, Silow $T$ et al. Body Awareness: a phenomenological inquiry into the common ground of mind-body therapies. Philos Ethics Humanit Med. 2011;6:6. DOI: http://dx.doi.org/10.1186/1747-5341-6-6

10. Stan DL, Collins NM, Olsen MM, Croghan I, Pruthi S. The Evolution of MindfulnessBased Physical Interventions in Breast Cancer Survivors. Evid Based Complement Alternat Med. 2012; 2012:758641.

11. Dropsy J. Leva i sin kropp. Stockholm: Aldus, 1975. 
12. Roxendal G. Body awareness therapy and the body awareness scale. Gothenburg: Dept. Rehabilitation Medicine, University of Gothenburg; 1985.

13. Malmgren-Olsson EB, Bränholm IB. A comparison between three physiotherapy approaches with regard to health-related factors in patients with non-specific musculoskeletal disorders. Dis Rehab 2002;24:308-317. DOI: http://dx.doi.org/10.1080/096382801100875 $\underline{02}$

14. Malmgren-Olsson E, Armelius B, Armelius K. A comparative outcome study of Body Awareness Therapy, Feldenkrais and conventional physiotherapy for patients with non-specific musculoskeletal disorders changes in psychological symptoms, pain and self-image. Physiother Theory Pract. 2001;17:77-95. DOI: http://dx.doi.org/10.1080/095939801750334 $\underline{167}$

15. Gard G. Body awareness therapy for patients with fibromyalgia and chronic pain. Disabil Rehabil. 2005;27:725-728. DOI: http://dx.doi.org/10.1080/096382804000090 $\underline{71}$

16. Ciconelli RM, Ferraz MB, Santos $W$, Meinão I, Quaresma MR. Tradução para a língua portuguesa e validação do questionário genérico de avaliação de qualidade de vida SF-36 (Brasil SF-36). Rev Bras Reumatol. 1999;39(3):143-152. DOI: http://dx.doi.org/10.1590/S0034-

\section{4}

17. Botega NJ, Bio MR, Zomignani MA, Garcia Jr C, Pereira WAB. Transtornos do humor em enfermaria de clínica médica e validação de escala de medida (HAD) de ansiedade e depressão. Rev Saúde Pública. 1995;29(5):359-363.

18. Leirvåg $H$, Pedersen $G$, Karterud S. Longterm continuation treatment after shortterm day treatment of female patients with severe personality disorders: Body awareness group therapy ver sus psychodynamic group therapy. Nord J Psychiatry. 2010;64:115-122.

19. Gyllensten AL, Skär L, Miller M, Gard G. Embodied identity - A deeper understanding of body awareness. Physiother Theory Pract. 2010;26(7):439-446, .

DOI: http://dx.doi.org/10.3109/095939809034229 $\underline{56}$

20. Hedlund L, Gyllensten AL. The physiotherapists' experience of Basic Body Awareness Therapy in patients with schizophrenia and schizophrenia spectrum disorders. J Bodywork Movem Therap. 2012;17(2):169-172. DOI: http://dx.doi.org/10.1016/j.jbmt.2012.10.00 $\underline{8}$

Recebido para publicação em 21/01/2014

Revisado em 04/04/2014

Aceito em 09/04/2014 